\section{Increased association of telomerase with short telomeres in yeast}

\author{
Alessandro Bianchi ${ }^{1}$ and David Shore \\ Department of Molecular Biology and National Centre \\ of Competence in Research (NCCR) "Frontiers in Genetics" \\ Program, University of Geneva, Sciences III, \\ CH-1211 Geneva 4, Switzerland
}

Telomere function is mediated by the assembly of a protein complex on an array of telomeric DNA (TG) repeats synthesized by the telomerase enzyme. Telomerase action at chromosome ends is finely tuned by the telomeric complex so that a constant average number of repeats is maintained. This is achieved through a negative feedback process that is sensitive to TG tract length, but whose underlying mechanism is unknown. We show that short telomeres, which are preferential substrates for telomerase, display increased association with the enzyme in the $S$ phase of the cell cycle, when telomerase acts. In addition, we provide support for a molecular mechanism by which this key step of telomerase recruitment is regulated by TG tract length.

Received April 27, 2007; revised version accepted June 5, 2007.

Telomeres promote genome integrity by reversing the natural erosion of terminal DNA sequences during cell division (the "end replication problem") and by preventing chromosome end exonucleolytic degradation or joining (de Lange 2005). In most eukaryotes this protective function is achieved via an array of tandemly arranged short terminal TG-rich repeats synthesized by the telomerase enzyme, a ribonucleoprotein with reverse transcriptase-like activity (Autexier and Lue 2006). The continued action of telomerase is required to ensure that the number of telomeric repeats hovers around an average species-specific value.

Although the presence of a functional telomerase enzyme is a necessary condition for telomere maintenance, it is not in itself sufficient. Genetic studies in budding yeast have uncovered several additional factors with an essential role in the telomerase pathway. For example, the telomerase-associated proteins Est1 and Est3, as well as a Cdc13, a DNA-binding protein with specificity for single-stranded telomeric TG repeats, are all required to promote telomerase (Est2) action (Lundblad and Szostak 1989; Nugent et al. 1996). In addition, the combined action of the Tell and Mecl kinases (orthologs of ATM [ataxia telangiectasia-mutated] and ATR [ATM and Rad3-related], respectively) is also required for telomerase-mediated telomere maintenance (Ritchie et al. 1999).

It has however become increasingly clear that several

[Keywords: Telomere length; telomerase regulation; cell cycle] ${ }^{1}$ Corresponding author.

E-MAIL Alessandro.Bianchi@molbio.unige.ch; FAX 41-22-379-6868. Article is online at http://www.genesdev.org/cgi/doi/10.1101/gad.438907. of the many proteins that comprise the telomeric complex act as negative regulators that prevent telomerase from acting at all telomeres during each cell cycle (Teixeira et al. 2004). A critical feature of the negative regulation of telomerase in cis at chromosome ends is the apparent ability of the telomeric complex to gauge telomere length based on the number of telomere repeatbound regulators (Rap1 and Rif proteins in yeast) (Marcand et al. 1997; Levy and Blackburn 2004). The telomeric complex then generates an inhibitory effect on telomerase whose strength increases with increasing TG tract length (Marcand et al. 1999). Although the general features of this feedback strategy of telomere length regulation have been proposed to apply to both yeasts and higher eukaryotes, the actual mechanism(s) by which the telomeric complex influences the action of telomerase and/or its positive regulators is largely unknown.

Based on available data, there are several possible explanations for telomere length-regulated activation of telomerase at chromosome ends, which can arguably be best summarized by three prevalent models (Fig. 1; for reviews, see Smogorzewska and de Lange 2004; Bertuch and Lundblad 2006). One model proposes that regulation of telomerase activity is achieved at the level of its association with the telomere (Fig. 1A). Telomerase recruitment has been shown to depend on a critical direct interaction between Est1 and Cdc13 (Evans and Lundblad 1999; Pennock et al. 2001; Bianchi et al. 2004). Because the single-stranded overhangs that serve as a substrate for Cdc13 loading might be formed equally at all telomeres (both long and short) (Wellinger et al. 1993), it is possible that a step subsequent to Cdc13 loading (which is presumed to be dependent on overhang formation) is subjected to regulation by TG tract length. In the model shown in Figure 1A, this regulated step is telomerase holoenzyme recruitment. An alternative view proposes that the association of telomerase with the telomere is not subjected to modulation by repeat array length, but rather that the enzyme's state is changed from inactive to active by telomere shortening. One incarnation of this latter model postulates that changes in the association of "activators" of telomerase, a role that has been proposed for Est1 (Evans and Lundblad 2002; Taggart et al. 2002; Singh and Lue 2003), would lead to changes in the activity, but not the telomere association, of the enzyme. A third possibility is that the amount of resection occurring in late $S$ phase at yeast telomeres, which is at least partially dependent on the MRX complex (Larrivee et al. 2004; Takata et al. 2005), might affect telomerase recruitment via the regulation of Cdc13 binding (Fig. 1C; Diede and Gottschling 2001; Takata et al. 2005).

\section{Results and Discussion}

The various models described above to explain the preferential activation of telomerase at shorter telomeres make different predictions with regard to the relative levels of association of the main players in the telomerase pathway at short versus normal-length telomeres (see Fig. 1, bottom, for an illustration of some of the existing possibilities). To attempt to discriminate between the models, we decided to employ a system that 
A Holoenzyme recruitment model

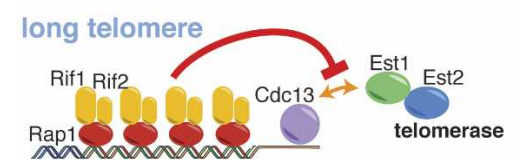

short telomere
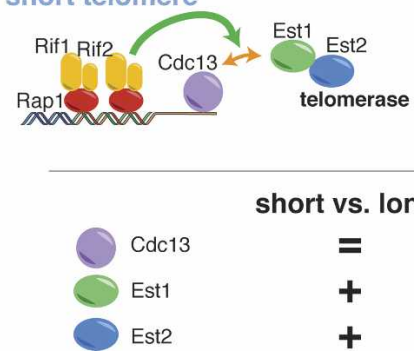

short vs. long

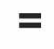

$+$
$+$
B

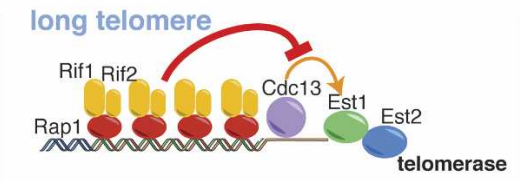

short telomere

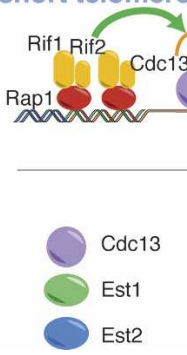

Est2

Figure 1. Molecular models leading to preferential elongation of shortened telomeres in yeast. (Top panels) Schematic representation of the association of selected telomeric proteins involved in the regulation of telomerase action with short and normal-length telomeres. Three different hypothetical models pertaining to possible mechanisms regulating the preferential activation of telomerase at short telomeres are illustrated. The regulated interaction between the Cdc13 and Est1 proteins is indicated by a double arrow ("activation" events are indicated throughout by simple green arrows, "repressive" events are indicated by red bars). In the bottom panels, the predicted enrichment at short versus normal-length telomeres for Cdc13, Est1, and Est2 is given.

allows the generation of a single shortened telomere in a yeast cell by site-specific (Cre-lox) recombination of a cassette that contains a telomere-proximal array of Rap1-binding sites (Fig. 2; Bianchi and Shore 2007). The presence of these sites leads to a terminal telomeric tract (beyond the distal loxP site) that is shortened by almost half, due to the inhibitory effect of Rapl on telomerase (Bianchi and Shore 2007; data not shown). Because the shortened telomere resulting from site-specific recombination is a preferred substrate for telomerase (Marcand et al. 1999; Bianchi and Shore 2007), this system offers the opportunity to characterize the molecular events that accompany telomerase action. We thus set out to analyze the protein composition, by chromatin immunoprecipitation (ChIP), of a shortened telomere located on chromosome V-R (chr V-R), in comparison with its unshortened counterpart in a companion strain where the loxP cassette did not include the Rap1-binding sites.

We first decided to investigate whether the association of telomerase itself is differentially regulated at the shortened telomere. Because the telomere binding of telomerase is cell cycle-regulated, we blocked the two strains (with shortened and normal-length chr V-R telomeres) in the G1 phase and then released them into a synchronous cell cycle. As previously described by others, we detected a (somewhat variable) association of Est2 in G1, and also in late S phase, which occurs at 70-80 min after release from the block under our experimental conditions (Fig. 3A; Taggart et al. 2002; Fisher et al. 2004; Schramke et al. 2004). Remarkably, the shortened telomere displayed significantly increased association of telomerase in S phase (Fig. 3A, right), the time when the enzyme acts at telomere ends, compared with the unshortened control. We then analyzed the telomere binding of the telomerase activators Est 1 and Cdc13, and found that Est1, like Est2, was preferentially associated with the short V-R telomere (Fig. 3B). In contrast, the association of Cdc13 with this telomere was not significantly changed upon its shortening (Fig. 3C). The relative enrichment at the shortened telomere for
Est 1 and Est2 (and lack of it for Cdc13) was very reproducible and found to occur in all experiments performed (see Fig. 3 legend for quantification and statistical analysis).

We confirmed the generality of these findings by quantifying the binding of both Est1 and Cdc13 to a second shortened telomere, this time on chr VII-L (Fig. 4). Because we had previously shown that the activation of a subtelomeric origin early in S phase takes place on this chromosome as a consequence of telomere shortening (Bianchi and Shore 2007), we inactivated this origin in order to make the binding profiles at the shortened and unshortened telomeres comparable in a quantitative manner. As expected, at this chromosome we also detected increased association for Est1, but not for Cdc13, with the shortened telomere (Fig. 4A,B, respectively). Thus, the relative differences in binding to telomeres of different lengths for Est1 and Cdc13 do not appear to be specific for any single chromosome end.

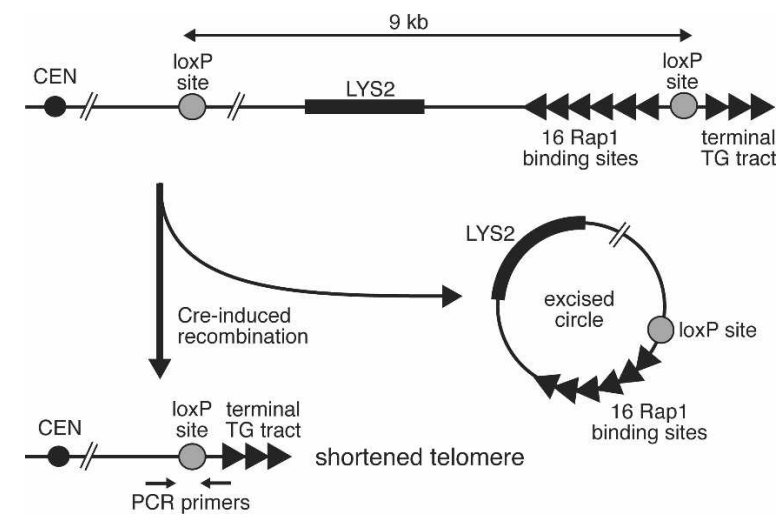

Figure 2. A system for the generation of a single shortened telomere on chr V-R. Schematic of the right telomere of chr V-R, generated by homologous recombination at the YER188W locus with a plasmid-borne cassette containing the indicated elements. The positions of the primers used for real-time PCR are shown. 
A

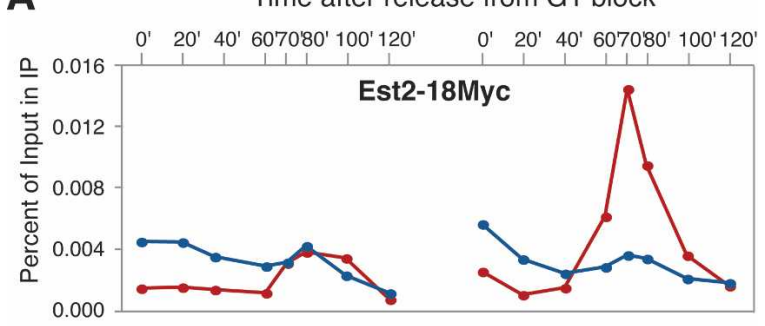

B

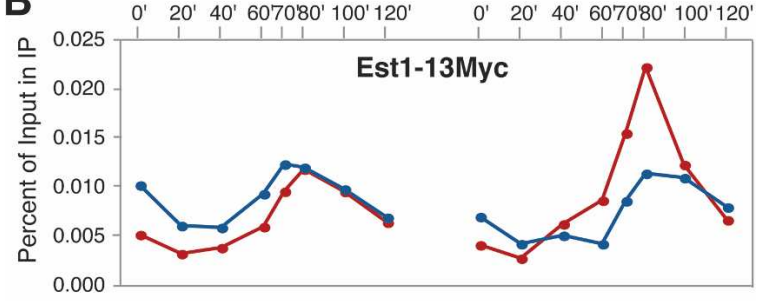

C

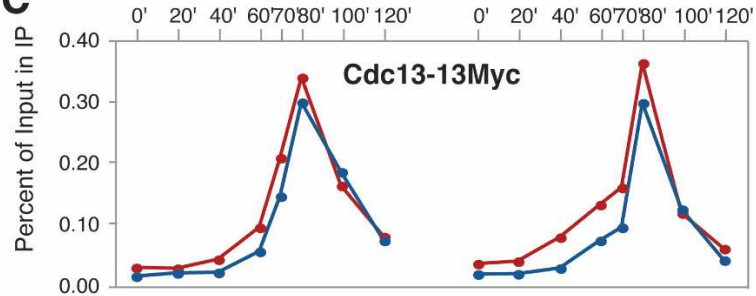

D

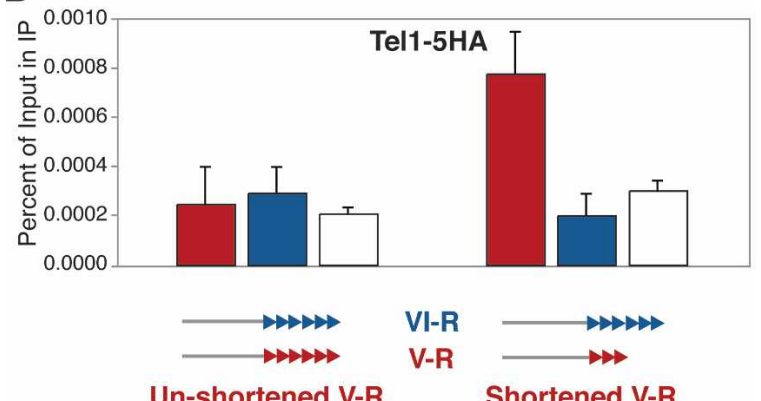

Un-shortened V-R Shortened V-R

Figure 3. Preferential recruitment of Est2, Est1, and Tel1, but not Cdc13, to a shortened telomere on chr V-R. Quantification of the association of Myc-tagged Est2 $(A)$, Est1 $(B)$, and Cdc13 $(C)$, and HA-tagged Tell $(D)$ with the recombined shortened (right), or unshortened (left) telomere at chr V-R (in red), and with the endogenous telomere on chr VI-R (in blue) by ChIP, on strains released synchronously from a block in $\mathrm{G} 1(A-C)$ or dividing asynchronously $(D)$. For $A-C$, quantification of the S-phase peak signal for the shortened versus unshortened V-R telomere, normalized for the signal obtained at the reference VI-R telomere, gave a value of 3.17 for Est2 $(1.69,2.14$, and 1.68 for three independent experiments; $P=0.044)$, 2.04 for Est1 (2.19 and 2.59 for two replicates; $P=0.009$ ), and 1.07 for Cdc13 (0.86 and 1.06, for two replicates; $P=0.886$ ). Histograms in $D$ represent averages and standard deviations of four independent asynchronously growing cultures (the white columns report association to the nontelomeric PDI1 locus). Student $t$-tests for V-R and VI-R, V-R and PDI1, and VI-R and PDIl yielded $P$ values of 0.677 , 0.616 , and 0.177 , respectively, for the strain with the unshortened telomeres, and $0.001,0.001$, and 0.122 , respectively, for the strain with the shortened telomeres.

The results described above are consistent with the notion that the recruitment of the telomerase enzyme to the telomere is a step regulated by telomere length and, more precisely, support a model in which the association of a telomerase holoenzyme (comprising Est1 and Est2) to the telomere is regulated (Fig. 1A). At the same time, our results do not support the idea that telomere shortening leads to telomerase "activation" or "relocalization" at the telomere, or that such effects are sufficient to explain preferential activation of the enzyme at shorter TG tracts (Fig. 1B). It has previously been shown that yeast telomerase is also telomere bound in the G1 phase of the cell cycle, in a Ku-dependent manner (Fisher et al. 2004). We did not observe an increase in the G1 association of telomerase at the shortened telomere, and this fact, together with the observation that the Est2 signal drops in late G1-early S, suggests that the aspect of telomerase association that is most relevant to the regulation of telomere length takes place at the time of telomerase action in late S phase. We propose that the increased Est2 (and Est1) signal at the shortened telomere is a reflection of a higher proportion of the telomeres having bound telomerase, rather than an increase in the number of telomerase molecules bound at each telomere, though we cannot formally exclude the possibility that a conformational change at the shortened telomere somehow leads to an increased ChIP signal for both Est2 and Est1.

The heightened binding of Estl with the shortened telomere is consistent with its proposed role as a telomerase holoenzyme component that helps to recruit the enzyme, but does not rule out some additional role in telomerase activation (Pennock et al. 2001; Taggart et al. 2002; Bianchi et al. 2004; Schramke et al. 2004). Impor-
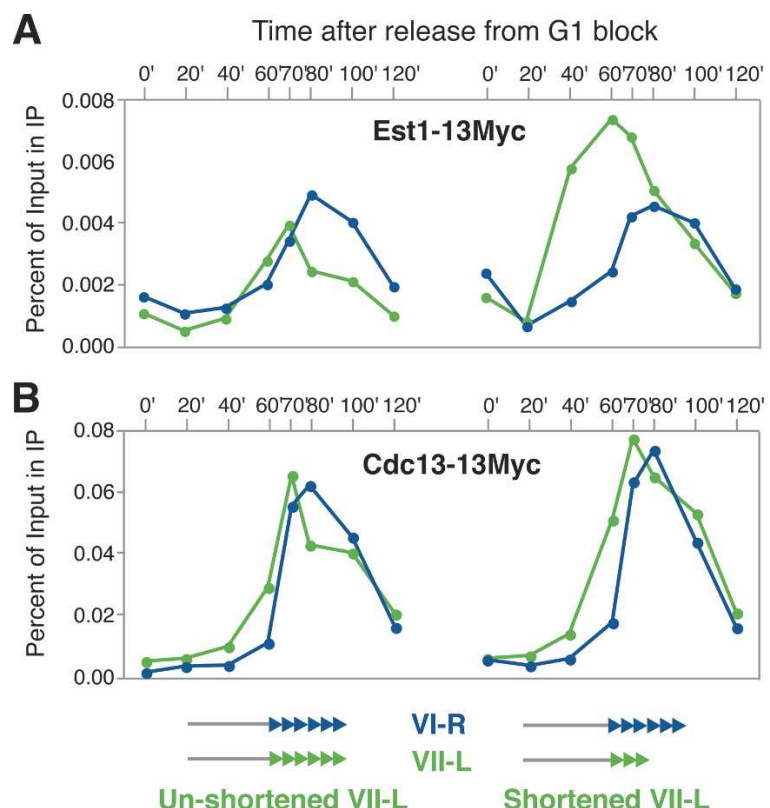

Figure 4. Preferential recruitment of Est1, but not Cdc13, to a shortened telomere on chr VII-L. Quantification of the association of Myc-tagged Est1 $(A)$ and $\mathrm{Cdc} 13(B)$ with the recombined shortened (right) or unshortened (left) telomere at chr VII-L (in green), and with the endogenous telomere on chr VI-R (in blue) by ChIP, on strains released synchronously from a block in G1. Quantification of the S-phase peak signal for the shortened versus unshortened VII-L telomere, normalized for the signal obtained at the reference VI-R telomere, gave a value of 2.04 for Est1 (and 1.93 for an independent experiment) and 1.00 for $\mathrm{Cdc} 13$ (and 1.27 for a replicate). In all of these strains, the region between coordinates 16308 and 18192 on chr VII, which contains a putative origin of DNA replication responsible for early replication of shortened telomeres, was deleted. 
tantly, the parallel increase in Est2 and Est1 association with the shortened telomere suggests that the interaction between Est1 and telomerase (through the telomerase RNA moiety) is not regulated by telomere length (Seto et al. 2002).

An alternative model (Fig. 1C), proposing that CA strand resection and TG overhang formation are responsible for increased Cdc13 recruitment (due to a longer binding substrate) and, ultimately, increased loading of telomerase and higher telomerase activity (Diede and Gottschling 2001; Takata et al. 2005; Negrini et al. 2007), is not supported by the finding that Cdc13 levels at the short and normal-length V-R and VII-L telomeres were unchanged. The absence of length-dependent modulation for Cdc13 contrasts markedly with the observed situation for Est1, suggesting that the interaction between these two proteins might represent the key step regulated by telomere length (Fig. 1A).

Recent work has identified serine residues in Cdc13 that are phosphorylated by Tel1/Mec1 (Tseng et al. 2006) in a domain of the protein shown to be involved in telomerase recruitment (Pennock et al. 2001; Bianchi et al. 2004). Because genetic evidence implicates these kinases, and particularly Tel1, in the regulation of telomere length (Ritchie et al. 1999), we decided to investigate the association of Tell with the shortened telomere. Even though we were unable to detect Tell binding to normal-length telomeres (where binding was indistinguishable in the ChIP assay from a nontelomeric control at the PDI1 locus), we found that association of the kinase with the shortened telomere was readily detectable, and significantly so over the unshortened telomeres (Fig. 3D). Although the association of Tell to normal-length telomeres has previously been reported to be limited to the G1 and G2/M phases of the cell cycle (Takata et al. 2004), we were unable to detect a signal at unshortened telomeres at any stage during the cell cycle whereas the protein was detectable at the shortened telomere throughout the cell cycle, including during $S$ phase, when the proposed interaction between Cdc13 and Est1 is thought to occur (data not shown). It thus remains unclear whether telomere shortening might lead to a change in the pattern of Tell telomere association during the cell cycle, apart from the observed quantitative change in binding.

Our results suggest that Tel1 (and possibly Mec1) phosphorylation activity might constitute the signal for the length-dependent association of telomerase with telomeres (Fig. 5). This model proposes that Cdc13 loading is not regulated by telomere length and that it is instead the ability of the protein to recruit telomerase that is the regulated step, likely via a modulation of its capability to interact with Est1. Based on our results with Tell and on the previous genetic and biochemical analysis of the proposed role of Tel1/Mec1 in phosphorylating Cdc13 (Tseng et al. 2006), we suggest that Tel1dependent phosphorylation is responsible for rendering Cdc13 competent for interaction with Est1 preferentially at shorter telomeres. The model is consistent with the observation that Tel1 (like Mre11, but unlike Mec1) is required for efficient Est2 and Est 1 recruitment to telomeres (Goudsouzian et al. 2006), and with the prediction, based on structural work, that Est1 is a phosphoserine-binding protein (Fukuhara et al. 2005). Taken together, these results point to telomere length-dependent binding of Tell to telomeres as a critical step in the

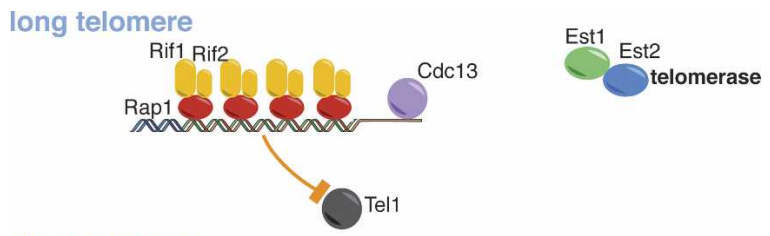

short telomere

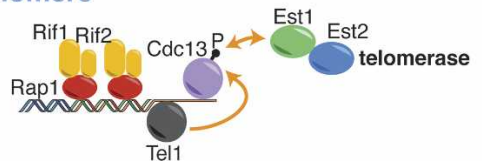

Figure 5. A model for a role of the Tell kinase in the length-dependent modulation of telomerase association with yeast telomeres. Schematic representation of the proposed model for Tell-dependent regulation of the preferential association of telomerase (and Est1) to short telomeres (see main text for details).

regulation of telomerase association with telomeres in S phase. Thus, the formerly proposed "closed" state of the telomere (Craven and Petes 1999; Teixeira et al. 2004), with regard to telomerase action, we suggest reflects decreased association of the enzyme with telomeres. Our biochemical analysis, combined with the available genetic data, indicates that a critical function of the telomere complex in operating a switch from a "closed" to "open" state is in regulating the recruitment, and therefore the activity at the telomere, of the Tell kinase.

\section{Materials and methods}

Strains and plasmids

Strains were in the W303 background lade2-1 his3-11,15 leu2-3,115 trp1-1 ura3-1 can1-100 RAD5) and carried a deletion of LYS2 and a PgalCre construct (pAB406) at the LEU2 locus: lys2::hphMX3 leu2::PgalCre. A TRP1-marked telomere at ADH4 (made with pAB556) was present in YAB1130-1172, and an ADE2-marked telomere at YER188W (made with pAB472) was present in YAB1023-1026. Tagging of Est1 and Cdc13 was obtained with PCR products generated from pFA6a-13MycHis3MX6 (Longtine et al. 1998), whereas tagging of Est2 was achieved with SwaI-cleaved pAB572, a derivative of pEST2Myc9-swiwel\#2, a generous gift of Timothy Fisher and Ginger Zakian (Fisher et al. 2004), and tagging of Tell was carried out with HindIII-cleaved pRS306/5HA-TEL1 (pAB548) a kind gift of Akira Matsuura (Takata et al. 2004). Plasmids pAB619 and pAB543 contained 16 inverted Rap1-binding sites adjacent to the distal loxP site (see Fig. 2), whereas pAB618 and pAB536 contained none. pAB618 and pAB619 targeted the ADH4 locus, whereas pAB536 and pAB543 targeted the YER188W locus. A cassette derived from pAB618 was present in YAB1130 (Fig. 3C, left), YAB1132 (Fig. 3B, left), YAB1167 (Fig. 3D, left), and YAB1171 (Fig. 3A, left); from pAB619 in YAB1131 (Fig. 3C, right), YAB1133 (Fig. 3B, right), YAB1168 (Fig. 3D, right), and YAB1172 (Fig. 3A, right); from pAB536 in YAB1023 (Fig. 4A, left) and YAB1025 (Fig. 4B, left); and from pAB543 in YAB1024 (Fig. 4A, right) and YAB1026 (Fig. 4B, right). In strains YAB1023-1026, the region between coordinates 16308 and 18192 on chr VII was deleted and replaced with a cloNAT cassette derived from pAG25 (Goldstein and McCusker 1999).

Induction of recombination and synchrony

Overnight cultures in $50 \mathrm{~mL}$ of SC medium lacking lysine and containing $2 \%$ raffinose were diluted into $350 \mathrm{~mL}$ of YPA plus $3 \%$ galactose. After $3-3.5 \mathrm{~h}$ at $30^{\circ} \mathrm{C}$ in galactose medium, cultures at a density of $1 \times 10^{7}$ cells per milliliter were blocked by treatment with $2 \mu \mathrm{M} \alpha$ factor for $130 \mathrm{~min}$ in YPAD at $30^{\circ} \mathrm{C}$. The arrest was removed by washing away the $\alpha$ factor and then cultures were released into a synchronous cell cycle by putting cells in YPAD medium at $18^{\circ} \mathrm{C}$.

\section{ChIP}

ChIP was performed as described previously (Bianchi et al. 2004; Fisher et al. 2004). Briefly, cells, after cross-linking in $1 \%$ formaldehyde, were 
lysed and sonicated. Immunoprecipitations were carried out with antiMyc 9E10 (Clontech) or anti-HA HA.11 (Covance) antibodies, and ProteinG Dynabeads (Dynal) against C-terminally 13Myc-tagged Est1 and Cdc13 proteins (Bianchi et al. 2004), 18Myc-tagged Est2 (Fisher et al. 2004), and 5HA-tagged Tell (Takata et al. 2004). Immunoprecipitates were washed with $0.2 \%$ SDS for Est 2 and Tel1, $0.25 \%$ SDS for Est1, and $0.3 \%$ SDS for Cdc13. Both an aliquot of sonicated cleared extract (input) and the immunoprecipitated material were de-cross-linked in TE plus $1 \%$ SDS for at least $8 \mathrm{~h}$ at $65^{\circ} \mathrm{C}$. Quantitation of immunoprecipitated DNA was obtained by real-time PCR using SYBR Green detection (Pfaffl 2001) on an Applied Biosystems HT7900 machine and software, and was expressed as percent of starting (input) material. Primers used were FSB2 (ACACCCACACACCACAGGAT) and RSB2 (GTTAACAGGCTGCG GCATT) for the recombined telomere at chr V-R and VII-L, FSB11 (CGT ATGCTAAAGTATATATTACTTCACTCCATT) and RSB11 (TCCGA ACTCAGTTACTATTGATGGAA) for the endogenous VI-R telomere, and FSB6 (AAACTCCGCAAGCACCAAGT) and RSB6 (CTGAAGACT CCGCTGTCGTTAA) for the internal PDI1 locus (in Fig. 4D).

\section{Acknowledgments}

We thank Thomas Kischlat and Heiko Schober for help with plasmid and strain construction, Susan Gasser for $\alpha$-factor, Timothy Fisher and Ginger Zakian for their ChIP protocol and for the Est2 tagging plasmid, Akira Matsuura for the Tell tagging plasmid, Nicolas Roggli for the artwork, and the members of the Shore laboratory. We are also extremely grateful to the NCCR Genomics Platform team in Geneva: Mylene Doquier, Didier Chollet, Christelle Barraclough, Patrick Descombes (and to Penelope!). A.B. received support from the Ligue Genevoise Contre le Cancer Funds were provided by grants from the Swiss National Science Foundation and the Swiss Cancer League (OncoSuisse), by the NCCR program "Frontiers in Genetics" (sponsored by the Swiss National Science Foundation), and by the Canton of Geneva. A.B. and D.S. designed the experiments and wrote the paper. A.B. performed the experiments.

\section{References}

Autexier, C. and Lue, N.F. 2006. The structure and function of telomerase reverse transcriptase. Annu. Rev. Biochem. 75: 493-517.

Bertuch, A.A. and Lundblad, V. 2006. The maintenance and masking of chromosome termini. Curr. Opin. Cell Biol. 18: 247-253.

Bianchi, A. and Shore, D. 2007. Early replication of short telomeres in budding yeast. Cell 128: 1051-1062.

Bianchi, A., Negrini, S., and Shore, D. 2004. Delivery of yeast telomerase to a DNA break depends on the recruitment functions of cdc13 and est1. Mol. Cell 16: 139-146.

Craven, R.J. and Petes, T.D. 1999. Dependence of the regulation of telomere length on the type of subtelomeric repeat in the yeast Saccharomyces cerevisiae. Genetics 152: 1531-1541.

de Lange, T. 2005. Shelterin: The protein complex that shapes and safeguards human telomeres. Genes \& Dev. 19: 2100-2110.

Diede, S.J. and Gottschling, D.E. 2001. Exonuclease activity is required for sequence addition and Cdc13p loading at a de novo telomere. Curr. Biol. 11: 1336-1340.

Evans, S.K. and Lundblad, V. 1999. Est1 and Cdc13 as comediators of telomerase access. Science 286: 117-120.

Evans, S.K. and Lundblad, V. 2002. The Est1 subunit of Saccharomyces cerevisiae telomerase makes multiple contributions to telomere length maintenance. Genetics 162: 1101-1115.

Fisher, T.S., Taggart, A.K., and Zakian, V.A. 2004. Cell cycle-dependent regulation of yeast telomerase by Ku. Nat. Struct. Mol. Biol. 11: 1198-1205.

Fukuhara, N., Ebert, J., Unterholzner, L., Lindner, D., Izaurralde, E., and Conti, E. 2005. SMG7 is a 14-3-3-like adaptor in the nonsense-mediated mRNA decay pathway. Mol. Cell 17: 537-547.

Goldstein, A.L. and McCusker, J.H. 1999. Three new dominant drug resistance cassettes for gene disruption in Saccharomyces cerevisiae. Yeast 15: 1541-1553.

Goudsouzian, L.K., Tuzon, C.T., and Zakian, V.A. 2006. S. cerevisiae Tellp and Mre1lp are required for normal levels of Est1p and Est2p telomere association. Mol. Cell 24: 603-610.

Larrivee, M., LeBel, C., and Wellinger, R.J. 2004. The generation of proper constitutive G-tails on yeast telomeres is dependent on the MRX complex. Genes \& Dev. 18: 1391-1396.

Levy, D.L. and Blackburn, E.H. 2004. Counting of Rif1p and Rif2p on Saccharomyces cerevisiae telomeres regulates telomere length. Mol. Cell. Biol. 24: 10857-10867.

Longtine, M.S., McKenzie III, A., Demarini, D.J., Shah, N.G., Wach, A., Brachat, A., Philippsen, P., and Pringle, J.R. 1998. Additional modules for versatile and economical PCR-based gene deletion and modification in Saccharomyces cerevisiae. Yeast 14: 953-961.

Lundblad, V. and Szostak, J.W. 1989. A mutant with a defect in telomere elongation leads to senescence in yeast. Cell 57: 633-643.

Marcand, S., Gilson, E., and Shore, D. 1997. A protein-counting mechanism for telomere length regulation in yeast. Science 275: 986-990.

Marcand, S., Brevet, V., and Gilson, E. 1999. Progressive cis-inhibition of telomerase upon telomere elongation. EMBO J. 18: 3509-3519.

Negrini, S., Ribaud, V., Bianchi, A., and Shore, D. 2007. DNA breaks are masked by multiple Rapl binding in yeast: Implications for telomere capping and telomerase regulation. Genes \& Dev. 21: 292-302.

Nugent, C.I., Hughes, T.R., Lue, N.F., and Lundblad, V. 1996. Cdc13p: A single-strand telomeric DNA-binding protein with a dual role in yeast telomere maintenance. Science 274: 249-252.

Pennock, E., Buckley, K., and Lundblad, V. 2001. Cdc13 delivers separate complexes to the telomere for end protection and replication. Cell 104: $387-396$

Pfaffl, M.W. 2001. A new mathematical model for relative quantification in real-time RT-PCR. Nucleic Acids Res. 29: e45.

Ritchie, K.B., Mallory, J.C., and Petes, T.D. 1999. Interactions of TLC1 (which encodes the RNA subunit of telomerase), TEL1, and MEC1 in regulating telomere length in the yeast Saccharomyces cerevisiae. Mol. Cell. Biol. 19: 6065-6075.

Schramke, V., Luciano, P., Brevet, V., Guillot, S., Corda, Y., Longhese, M.P., Gilson, E., and Geli, V. 2004. RPA regulates telomerase action by providing Estlp access to chromosome ends. Nat. Genet. 36: 4654.

Seto, A.G., Livengood, A.J., Tzfati, Y., Blackburn, E.H., and Cech, T.R. 2002. A bulged stem tethers Estlp to telomerase RNA in budding yeast. Genes \& Dev. 16: 2800-2812.

Singh, S.M. and Lue, N.F. 2003. Ever shorter telomere 1 (EST1)-dependent reverse transcription by Candida telomerase in vitro: Evidence in support of an activating function. Proc. Nat1. Acad. Sci. 100: 57185723.

Smogorzewska, A. and de Lange, T. 2004. Regulation of telomerase by telomeric proteins. Annu. Rev. Biochem. 73: 177-208.

Taggart, A.K., Teng, S.C., and Zakian, V.A. 2002. Est1p as a cell cycleregulated activator of telomere-bound telomerase. Science 297: 10231026.

Takata, H., Kanoh, Y., Gunge, N., Shirahige, K., and Matsuura, A. 2004. Reciprocal association of the budding yeast ATM-related proteins Tell and Mecl with telomeres in vivo. Mol. Cell 14: 515-522.

Takata, H., Tanaka, Y., and Matsuura, A. 2005. Late S phase-specific recruitment of Mre11 complex triggers hierarchical assembly of telomere replication proteins in Saccharomyces cerevisiae. Mol. Cell 17: 573-583.

Teixeira, M.T., Arneric, M., Sperisen, P., and Lingner, J. 2004. Telomere length homeostasis is achieved via a switch between telomeraseextendible and -nonextendible states. Cell 117: 323-335.

Tseng, S.F., Lin, J.J., and Teng, S.C. 2006. The telomerase-recruitment domain of the telomere binding protein Cdc13 is regulated by Meclp/ Tellp-dependent phosphorylation. Nucleic Acids Res. 34: 63276336.

Wellinger, R.J., Wolf, A.J., and Zakian, V.A. 1993. Saccharomyces telomeres acquire single-strand TG1-3 tails late in S phase. Cell 72: 5160. 


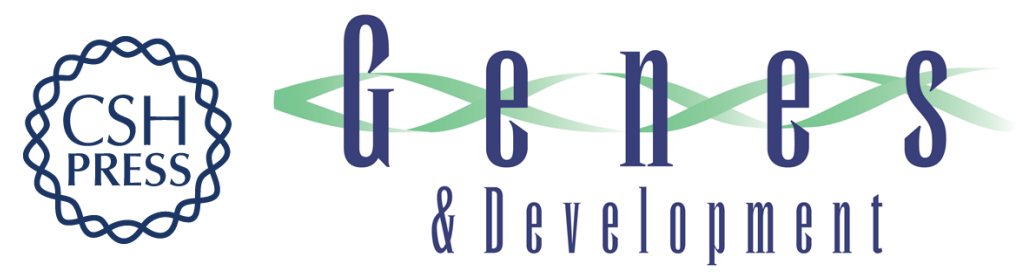

\section{Increased association of telomerase with short telomeres in yeast}

Alessandro Bianchi and David Shore

Genes Dev. 2007, 21:

Access the most recent version at doi:10.1101/gad.438907

References This article cites 34 articles, 14 of which can be accessed free at: http://genesdev.cshlp.org/content/21/14/1726.full.html\#ref-list-1

License

Email Alerting Receive free email alerts when new articles cite this article - sign up in the box at the top Service right corner of the article or click here.

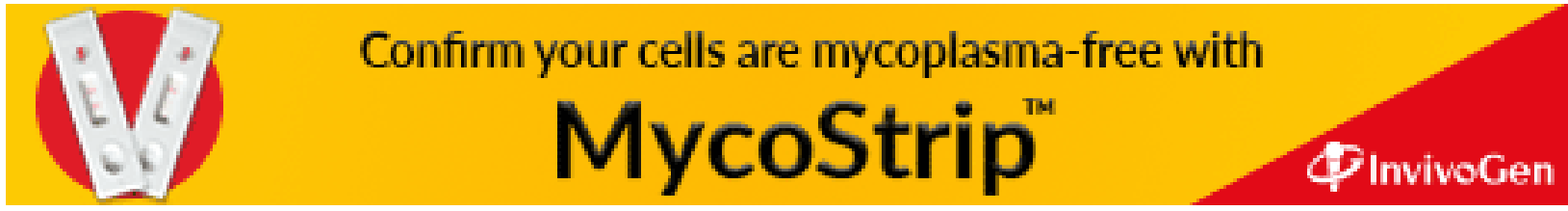

